\title{
Safety, Tolerability and Efficacy of Eslicarbazepine Acetate as Adjunctive Therapy in Patients Aged $\geq 65$ Years with Focal Seizures
}

\author{
Raquel Costa ${ }^{1} \cdot$ Bernhard Steinhoff $^{2} \cdot$ Helena Gama ${ }^{1} \cdot$ Fábio Ikedo ${ }^{1} \cdot$ José-Francisco Rocha ${ }^{1}$. \\ Patrício Soares-da-Silva ${ }^{1,3,4}$
}

Published online: 1 November 2018

(c) The Author(s) 2018

\begin{abstract}
Background The incidence of epilepsy is high within the first few years of life, stabilizes over the second through fifth decades, and then rises again. Treatment of elderly patients with antiepileptic drugs (AEDs) is complicated by increased sensitivity to drug effects, altered pharmacokinetics and an increased risk for drug interactions due to polytherapy. On the other hand, the safety and efficacy data of AEDs attained during clinical development programmes are relatively limited for this age group. Objective The aim of this study was to evaluate the safety, tolerability and efficacy of eslicarbazepine acetate (ESL) as adjunctive therapy in patients aged $\geq 65$ years with focal-onset seizures (FOS).

Methods This was an international, multicentre, open-label, non-controlled, single-arm, post-European approval commitment study with flexible doses of ESL between 400 and $1200 \mathrm{mg} /$ day. Seventy-two elderly patients with at least two FOS in the prior 4 weeks, and treated with one or two AEDs, were enrolled. The study consisted of an 8-week baseline, followed by a 26-week treatment period during which the investigator was allowed to up- or down-titrate the ESL dose, and a 4-week follow-up period. Safety and tolerability were assessed as well as mental sedation, cognitive mental state and suicidal ideation. Efficacy was assessed based on patient diaries regarding the absolute and relative changes in seizure frequency, change in intellectual impairment and quality of life.

Results Overall, 47 (65.3\%) patients experienced 152 treatment-emergent adverse events (TEAEs). The most frequent were dizziness (12.5\%), somnolence (9.7\%), fatigue, convulsion and hyponatraemia (8.3\% each). All patients that experienced hyponatraemia (6/72) recovered without sequelae. Three patients died during the study (due to cardiac failure, glioblastoma multiforme and ischaemic stroke, all considered unrelated to ESL). Overall, 16 (22.2\%) patients discontinued prematurely due to TEAEs. The incidences of clinically significant findings were low for vital signs, ECG, physical and neurological examinations. No TEAEs of hypothyroidism were reported; however, 24 (33.3\%) patients presented post-baseline shifts from normal to decreased free T4 levels (not clinically significant). ESL decreased standardized seizure frequency from a mean of 4.8 seizures at baseline to 3.6 seizures at endpoint $(p>0.05)$; and mean number of days with seizures significantly decreased from 4.1 (baseline) to 2.8 at endpoint $(p=0.0408)$.

Conclusion ESL taken once daily (400-1200 mg) as adjunctive therapy in patients aged $\geq 65$ years was found to be safe, well tolerated and efficacious (EudraCT number: 2009-012587-14).
\end{abstract}

Electronic supplementary material The online version of this article (https://doi.org/10.1007/s40266-018-0602-y) contains supplementary material, which is available to authorized users.

Patrício Soares-da-Silva

psoares.silva@bial.com

Extended author information available on the last page of the article

\section{Key Points}

Use of eslicarbazepine acetate (ESL) in elderly patients was safe, well tolerated and effective in reducing seizure frequency.

The most common treatment-emergent adverse events (TEAEs) were dizziness, somnolence, fatigue, convulsion and hyponatraemia.

No relationship was observed between the incidence or severity of hyponatraemia and ESL dose. 


\section{Introduction}

Epilepsy affects more than 50 million adults and children worldwide. The two highest peaks of incidence are in children and in the elderly population aged $>65$ years. Prevalence estimates in the total population vary from 4 to 8 per 1000 subjects [1]. Focal-onset seizures (FOS), which are related to a focal brain dysfunction, are the most frequent type ( $\sim 60 \%$ of cases). Antiepileptic drugs (AEDs) are the major therapeutic intervention and approximately $60 \%$ of newly diagnosed patients become seizure-free on a single AED; but in about $30 \%$ of patients, seizures are not satisfactorily controlled and many patients have significant adverse events [1]. This lack of seizure control means that combination therapy is often recommended, but a sizeable proportion of patients continue to have seizures regularly despite therapy with more than one AED. Therefore, there is still a need for new, effective AEDs, particularly those that can be used safely as adjuncts to standard therapy, in order to further reduce seizure frequency.

Epidemiological studies of several populations have found that the incidence of seizures, epilepsy, or both, is high within the first few years of life, stabilizes over the second through fifth decades, and then rises again. The elderly is the most rapidly growing segment of the population, and incidence of epilepsy is higher in this age group than in any other. Treatment of older patients with AEDs, as with many other medications, is complicated by increased sensitivity to drug effects, altered pharmacokinetics, and an increased risk for drug interactions due to multiple drug therapy [1-3]. Additionally, there are relatively limited data to guide the use of anti-epileptic medications and other treatments in this patient population [3].

Eslicarbazepine acetate (ESL) is a voltage-gated sodium channel blocker currently approved by the European Medicines Agency (EMA), the United States Food and Drug Administration (FDA) and Health Canada, as adjunctive and monotherapy in adults for the treatment of FOS with or without secondary generalization. Paediatric indication was granted by the EMA and Health Canada as adjunctive therapy in adolescents and children aged $>6$ years with FOS with or without secondary generalization. ESL was also approved by the FDA as adjunctive and monotherapy in children 4 years of age and older.

ESL is chemically related to carbamazepine and oxcarbazepine, but contains molecular variations that result in differences in metabolism [4]. These metabolic differences may result in a lower potential for drug interactions, good tolerability, once-daily dosing and a simple titration regimen. In humans, eslicarbazepine ( $S$-licarbazepine, BIA 2-194), is the major active metabolite of ESL and the entity responsible for the pharmacological effect [5]. Steady-state of eslicarbazepine plasma concentration occurs after 4 or 5 days of once-daily dosing [6, 7]. Pharmacokinetics is dependent on renal function [8], but is not significantly affected by the presence of food [9], moderate hepatic impairment [10], age [7] or gender [6].

This clinical study was a post-approval commitment with the EMA and aimed to assess the safety, tolerability and efficacy of ESL as adjunctive therapy for focal-onset seizures in elderly patients (aged 65 years or older).

\section{Methods}

\subsection{Study Design}

This open-label study (trial registration EudraCT No. 2009012587-14) was conducted in 44 centres across 13 countries in Europe and Asia (2 in Austria, 5 in Bulgaria, 2 in Croatia, 7 in the Czech Republic, 4 in Germany, 3 in Malaysia, 3 in Poland, 2 in Portugal, 3 in Romania, 3 in South Korea, 4 in Spain, 2 in Taiwan and 4 in Thailand), to evaluate the safety, tolerability and efficacy of ESL as adjunctive therapy in patients aged $\geq 65$ years with FOS.

The study design consisted of an 8-week baseline period, followed by a 26-week treatment period and a 4-week follow-up period. Patients were treated in the range of 400-1200 mg ESL and the recommended starting dose was $400 \mathrm{mg}$ once daily for 2 weeks. Thereafter, the investigator was allowed to up- or down-titrate the ESL dose in 400-mg steps based on individual response, including safety issues. During the 4-week follow-up period, if needed, ESL could be tapered off, also in $400 \mathrm{mg}$ steps, and a standard AED could be introduced. The investigator was also allowed to introduce, remove, up- or down-titrate any other AED.

ESL was administered orally, once daily (QD), at approximately the same time each day, preferably in the evening or before going to bed. The ESL $400 \mathrm{mg}$ and $800 \mathrm{mg}$ divisible tablets used in the study were manufactured by BIALPortela \& C $C^{\text {a }}$ S.A., Coronado (S. Romão e S. Mamede), Portugal. The sample size was discussed and agreed with the EMA. A study population of 100 evaluable patients aged $\geq 65$ years was considered adequate. However, due to recruitment difficulties, the sponsor agreed with the EMA to stop recruitment when 72 patients had been enrolled.

The schedule of assessments in this study included the following timing points: (1) screening visit (V1), 8 weeks \pm 3 days before V2; (2) baseline visit (V2); (3) treatment period visit (V3), 2 weeks \pm 3 days after V2; (4) telephone contact, 2 weeks \pm 3 days after V3; (5) treatment period visit (V4), 4 weeks \pm 3 days after V3; (6) treatment period visit (V5), 8 weeks \pm 3 days after V4; (7) end-ofstudy visit (V6) or early discontinuation visit (EDV), 
12 weeks \pm 3 days after V5; and (8) follow-up visit (V7), 4 weeks \pm 3 days after V6/EDV.

Descriptive statistics were calculated for demographic data, type of epilepsy, seizure types and frequency of side effects. Changes in seizure frequency were conducted using Chi square tests or Fisher exact tests ( $p$ value $<0.05$ was considered as significant).

The study was approved by the national Independent Ethics Committee and Competent Authorities. The study was conducted according to the Helsinki Declaration, ICH Good Clinical Practice recommendations and applicable local regulations. Written informed consent was obtained for each study participant.

\subsection{Population}

Adult men or women (aged 65 years or older) were eligible if they had a documented diagnosis of epilepsy for at least 12 months and at least two focal seizures (including subtypes of focal aware seizures-focal impaired awareness seizures evolving to secondarily generalized) in the 4 weeks prior to enrolment, and were currently being treated with one or two AEDs (any except oxcarbazepine)—vagus nerve stimulation (VNS) was considered an AED - in a stable regimen for at least 4 weeks prior to enrolment. Patients with only focal aware seizures with no motor symptomatology, primarily generalized seizures, known progressive neurological disorders and progressive dementia, history of status epilepticus or cluster seizures within the 3 months prior to enrolment were excluded. Patients with seizures of non-epileptic origin, major psychiatric disorders, history of suicide attempt, any uncontrolled disorder, clinical laboratory abnormalities as defined by the investigator and calculated creatinine clearance $<30 \mathrm{~mL} / \mathrm{min}$ were also excluded. A guidance was given to the investigator regarding exclusion values for laboratory abnormalities such as plasma sodium $<130 \mathrm{mmol} / \mathrm{L}$, alanine or aspartate aminotransferases $>2.0$ times the upper limit of the normal range, or white blood cell count $<3000$ cells/ $\mathrm{mm}^{3}$.

\subsection{Safety Assessments}

Safety and tolerability were assessed through incidence of treatment-emergent adverse events (TEAEs), laboratory evaluations, vital signs, 12-lead ECG and physical/neurological examinations. Mental sedation and intellectual impairment were assessed at baseline (V2), V5 and V6/EDV using Norris' adapted method, four 10-point numeric rating scales. This is a numeric version of a series of 16 analogue scales described by Norris [11]. The 16 items are divided into four categories of effects ('mental sedation and intellectual impairment', 'physical sedation and bodily impairments', 'tranquilization and calming effects', and 'other types of feelings and attitudes'). The four items used in this study were the ones associated with the category 'mental sedation or intellectual impairment'.

Intellectual ability was also assessed at last visit (V6/ EDV) using the Patient's Global Impression of Change (PGIC) of Intellectual Ability, a 7-point rating scale. The cognitive mental state of patients was examined only at screening (V1) by means of the Mini-Mental State Examination (MMSE) questionnaire. Suicidal ideation and behaviour was assessed at baseline, V3, V4, V5, V6/EDV and V7 using the Columbia-Suicide Severity Rating Scale (C-SSRS).

\subsection{Efficacy Assessments}

The Full Analysis Set (FAS) consisted of all patients who received at least one dose of treatment and had at least one day of efficacy evaluation reported after baseline. The Safety Set (SS) consisted of all subjects who had received at least one dose of ESL. The Per-Protocol (PP) set consisted of all patients in the FAS who completed the treatment period and did not have any protocol deviation in a sufficiently serious manner to warrant data (but not patient) exclusion. Decisions regarding data exclusion from the PP set were taken prior the start of the statistical analyses in a data review meeting.

The efficacy assessments were based on occurrence (date and time) and type of seizures recorded in the patient diaries. Quality of Life in Epilepsy-31 inventory (QOLIE-31) performed at baseline, V4 and V6/EDV, and PGIC of intellectual ability performed at last visit (V6/EDV), were also applied.

\section{Results}

\subsection{Population}

A total of 72 patients were allocated to study treatment and included in the SS. Of these, 50 (69.4\%) completed the treatment period, while $22(30.6 \%)$ prematurely terminated the trial. The most frequent reason for premature trial termination was the occurrence of an adverse event (16 patients, $22.2 \%$ ).

Patients' age ranged from 65 to 84 years, with a mean (SD) age of $71.6(5.40)$ years. The patients aged 65-69 years comprised $41.7 \%$ of the SS, those aged 70-74 years and $75-79$ years were $26.4 \%$ (each group), whereas the 80 - to 84 -year-old patients comprised $5.6 \%$ of the population. Male patients accounted for $52.8 \%$. The majority of patients (83.3\%) were Caucasian.

The mean epilepsy duration was 246.8 months, ranging from 12.2 to 850 months. The most frequently reported epilepsy aetiologies were 'other or unknown' (38.9\% of 
patients), cerebrovascular disease $(23.6 \%)$ and cranial trauma or injuries $(11.1 \%)$, and $93.1 \%$ of the patients had at least one concomitant disease at screening. The most common concomitant diseases (in $\geq 5 \%$ ) at baseline were hypertension (54.2\%), dyslipidaemia (13.9\%), obesity (13.9\%), hypercholesterolaemia $(11.1 \%)$, myocardial ischaemia $(11.1 \%)$, benign prostatic hyperplasia (9.7\%), depression (9.7\%), insomnia (8.3\%), hypothyroidism $(8.3 \%)$, back pain (6.9\%), and hypoacusis, decreased creatinine clearance, osteoarthritis, meningioma, cerebrovascular disorder, hemiparesis, and arteriosclerosis (5.6\%, each).

The most frequently used concomitant AEDs were valproic acid (36.1\% of patients), carbamazepine $(31.9 \%)$, lamotrigine $(25.0 \%)$ and levetiracetam (13.9\%). Concomitant non-AED medication was taken by $83.3 \%$ of patients. Regarding the use of concomitant drugs (in more than one subject) that have been associated with hyponatraemia, indapamide was used by six patients $(8.3 \%)$, hydrochlorothiazide, furosemide and spironolactone by three subjects $(4.2 \%)$ and amiloride, hydrochlorothiazide and paroxetine were used by two patients $(2.8 \%)$.

The MMSE was performed at V1 and the vast majority of patients had either normal cognitive function (68.1\%) or mild cognitive impairment (26.4\%). Severe cognitive impairment was reported for one subject (1.4\%).

\subsection{Safety Results}

Mean treatment duration was 151.8 days and the mean ESL dose during the overall treatment period was $591.9 \mathrm{mg} /$ day. The majority of patients were exposed to doses not higher than $800 \mathrm{mg}$ QD. An ESL dose of $1200 \mathrm{mg}$ QD was used by 10 patients ( $13.9 \%$ of the SS).

TEAEs, reported by $\geq 5 \%$ of patients, were dizziness $(12.5 \%)$, somnolence $(9.7 \%)$, fatigue, convulsion and hyponatraemia ( $8.3 \%$ each), nasopharyngitis $(6.9 \%)$ and upper respiratory tract infection $(5.6 \%)$ (Table 1). The majority of TEAEs were assessed by the investigators as mild (36.1\%) to moderate (34.7\%) (Table 1). In total, 16 treatment-emergent serious adverse events (TESAEs) were reported for 10 patients (13.9\%). No TESAE occurred in more than one subject (Table 2). Three patients died (4.2\%), one due to cardiac failure, one due to glioblastoma multiforme and one due to ischaemic stroke. None of the fatal cases were considered related to study medication, either by the investigator or by the sponsor. Sixteen $(22.2 \%)$ patients prematurely discontinued the study because of TEAEs. The TEAEs leading to the discontinuation of more than one subject were hyponatraemia (3 patients), dizziness and fatigue (2 patients each). The lowest value of sodium blood measured was $120 \mathrm{mmol} / \mathrm{L}$ and all patients that experienced hyponatraemia (6/72) recovered.
Of the total number of patients exposed to ESL $400 \mathrm{mg}$ $(n=68), 31(45.6 \%)$ experienced at least one TEAE while on this dose. The most commonly reported TEAEs (occurring in $\geq 3$ patients) were fatigue ( 6 patients), dizziness, somnolence, nasopharyngitis ( 5 patients each), convulsion and hyponatraemia (4 patients each). For the ESL 800-mg dose, $21(50 \%)$ of the 42 exposed patients experienced at least one TEAE while on this dose, with the most common being dizziness and blood creatine phosphokinase (CPK) increase (3 patients each). For the ESL 1200-mg dose, only four TEAEs were reported among three (30\%) of the ten exposed patients (abdominal pain upper, prostate cancer, anxiety and urinary retention). The pattern and incidence of TEAEs was overall similar between gender and age groups, although the small number of analysed patients does not allow meaningful comparisons (see Table 1 and Table 2, respectively, in the electronic supplementary material).

Laboratory TEAEs affecting more than one subject were hyponatraemia (6 patients, 8.3\%), blood CPK increased (3 patients, $4.2 \%$ ) and gamma-GT increased (3 patients, $4.2 \%$ ) (Table 1). Overall, $19.4 \%$ of patients experienced a sodium decrease from baseline $\geq 10 \mathrm{mmol} / \mathrm{L}$. Post-baseline incidences of sodium levels $<130$ and $<125 \mathrm{mmol} / \mathrm{L}$ were $9.7 \%$ and $1.4 \%$, respectively. Of the 10 subjects with sodium levels $<130 \mathrm{mmol} / \mathrm{L}$, five were receiving ESL $400 \mathrm{mg}$ and five ESL $800 \mathrm{mg}$. The single subject with sodium $<125 \mathrm{mmol} / \mathrm{L}$ was receiving ESL $400 \mathrm{mg}$.

For thyroid function tests, although there were no TEAEs concerning hypothyroidism reported during the study, 24 patients $(33.3 \%)$ presented post-baseline shifts from normal to low free T4 levels at any post-baseline assessment. There were no patients presenting a shift from normal to a clinically significant value for any thyroid parameter per investigators assessment. One subject had a shift from normal to clinically important free T3 low value per sponsor's criteria. No relevant changes were observed for liver function tests.

The incidences of relevant findings for vital signs, ECG, and physical and neurological examinations were low, with no trends observed. The only significant new onset post-baseline finding from the 12-lead ECG was one case of atrial fibrillation, assessed as not related to study treatment. Rash was reported in two patients (2.8\%), but neither were assessed as severe or serious.

Norris' adapted mental sedation scales showed minor changes from baseline to last study visit in subject responses towards a slight worsening of mean values (see Table 3 in the electronic supplementary material). These results did not correlate with the subjects' intellectual ability ratings by means of the PGIC score: approximately half of the patients reported improvements, compared with $16.9 \%$ of patients who considered values to have worsened (see Table 4 in the electronic supplementary material). 
Table 1 Summary of treatment-emergent adverse events (TEAEs) reported for the safety population $(N=72)$

\begin{tabular}{|c|c|c|}
\hline TEAEs & $\begin{array}{l}\text { No. of patients ( } \% \text { of } \\
\text { safety population) }\end{array}$ & $\begin{array}{l}\text { Number } \\
\text { of TEAEs }\end{array}$ \\
\hline All & $47(65.3)$ & 152 \\
\hline \multicolumn{3}{|c|}{ Frequency of patients with TEAEs $\geq 5 \%$, categorized by preferred term } \\
\hline Dizziness & $9(12.5)$ & 11 \\
\hline Somnolence & $7(9.7)$ & 7 \\
\hline Fatigue & $6(8.3)$ & 7 \\
\hline Convulsion & $6(8.3)$ & 6 \\
\hline Hyponatraemia & $6(8.3)$ & 6 \\
\hline Nasopharyngitis & $5(6.9)$ & 5 \\
\hline Upper respiratory tract infection & $4(5.6)$ & 5 \\
\hline \multicolumn{3}{|c|}{ Frequency of patients with at least one TEAE, categorized by severity } \\
\hline Mild & $26(36.1)$ & 67 \\
\hline Moderate & $25(34.7)$ & 44 \\
\hline Severe & $12(16.7)$ & 18 \\
\hline Not applicable ${ }^{a}$ & $14(19.4)$ & 23 \\
\hline \multicolumn{3}{|c|}{ Frequency of patients with at least one TEAE, categorized by System Organ Class-Investigations } \\
\hline Blood creatine phosphokinase increased & $3(4.2)$ & 3 \\
\hline Gamma-glutamyltransferase increased & $3(4.2)$ & 3 \\
\hline Blood alkaline phosphatase increased & $1(1.4)$ & 1 \\
\hline Blood potassium decreased & $1(1.4)$ & 1 \\
\hline Blood urea increased & $1(1.4)$ & 1 \\
\hline White blood cell count decreased & $1(1.4)$ & 1 \\
\hline \multicolumn{3}{|c|}{ Frequency of patients with at least one TEAE, categorized by System Organ Class-Metabolism and nutrition disorders } \\
\hline Hyponatraemia & $6(8.3)$ & 6 \\
\hline Hyperglycaemia & $1(1.4)$ & 2 \\
\hline Hyperkalaemia & $1(1.4)$ & 1 \\
\hline Hyperlipidaemia & $1(1.4)$ & 1 \\
\hline Hypertriglyceridaemia & $1(1.4)$ & 1 \\
\hline Hypokalaemia & $1(1.4)$ & 1 \\
\hline
\end{tabular}

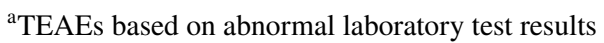

No reports of suicidality (suicidal ideation or behaviour) were observed post-baseline, as assessed by TEAEs and the C-SSRS.

\subsection{Efficacy Results}

The mean (SD) standardized seizure frequency decreased from 4.8 (5.53) during the 8-week baseline period to 3.6 (5.84) during the 26-week treatment period (FAS) $(p=0.1824)$. Although not statistically significant $(p \geq 0.05$ ), improvements were observed for all types of seizures throughout the 26-week treatment period. By type of seizure, the mean (SD) standardized frequency decreased from 3.6 (5.43) to 3.1 (5.00) for focal aware seizures with motor component $(p=0.6883)$, from 3.5
(3.77) to 2.6 (4.11) for focal impaired awareness seizures with motor component $(p=0.2105)$, and from $1.6(2.03)$ to 0.6 (1.11) for focal evolving to secondarily generalized seizures $(p=0.0594)$.

During the treatment period, $11(15.5 \%)$ patients were seizure free and a total of $39(54.9 \%)$ patients were responders; that is, reported a $\geq 50 \%$ reduction of seizure frequency relative to baseline (Table 3 ). Similar results were obtained when the PP set was considered.

The mean (SD) number of days with seizures statistically significantly decreased from 4.1 (3.72) during the baseline period to 2.8 (3.97) during the treatment period $(p=0.0408)$.

Minimal changes were observed for all QOLIE-31 scores throughout the treatment period. Although not 
Table 2 Serious treatment-emergent adverse events (TEAEs) reported for safety population $(N=72)$

\begin{tabular}{|c|c|c|}
\hline Primary System Organ Class preferred term & No. of patients (\% of safety population) & $\begin{array}{l}\text { Number } \\
\text { of TEAEs }\end{array}$ \\
\hline Patients with any serious TEAE & $10(13.9)$ & 16 \\
\hline Nervous system disorders & $5(6.9)$ & 5 \\
\hline Altered state of consciousness ${ }^{\mathrm{a}}$ & $1(1.4)$ & 1 \\
\hline Grand mal convulsion ${ }^{\mathrm{a}}$ & $1(1.4)$ & 1 \\
\hline Ischaemic stroke & $1(1.4)$ & 1 \\
\hline Lacunar infarction & $1(1.4)$ & 1 \\
\hline Presyncope & $1(1.4)$ & 1 \\
\hline Infections and infestations & $3(4.2)$ & 3 \\
\hline Bronchitis & $1(1.4)$ & 1 \\
\hline Gastroenteritis & $1(1.4)$ & 1 \\
\hline Pneumonia & $1(1.4)$ & 1 \\
\hline Cardiac disorders & $2(2.8)$ & 2 \\
\hline Cardiac failure & $1(1.4)$ & 1 \\
\hline Coronary artery disease & $1(1.4)$ & 1 \\
\hline Neoplasms benign, malignant and unspecified & $2(2.8)$ & 2 \\
\hline Glioblastoma multiforme & $1(1.4)$ & 1 \\
\hline Prostate cancer & $1(1.4)$ & 1 \\
\hline Injury, poisoning and procedural complications & $1(1.4)$ & 1 \\
\hline Toxicity to various agents ${ }^{\mathrm{a}}$ & $1(1.4)$ & 1 \\
\hline Metabolism and nutrition disorders & $1(1.4)$ & 1 \\
\hline Hyponatraemia $^{\mathrm{a}}$ & $1(1.4)$ & 1 \\
\hline Psychiatric disorders & $1(1.4)$ & 1 \\
\hline Postictal psychosis & $1(1.4)$ & 1 \\
\hline Respiratory, thoracic and mediastinal disorders & $2(2.8)$ & 2 \\
\hline Upper airway obstruction & $1(1.4)$ & 1 \\
\hline Gastrointestinal disorders & $1(1.4)$ & 1 \\
\hline Gastritis & $1(1.4)$ & 1 \\
\hline
\end{tabular}

${ }^{a}$ Serious adverse events considered at least possibly related to study treatment

Table 3 Summary of efficacy data during treatment period

\begin{tabular}{lcr}
\hline & $\begin{array}{l}\text { Full analysis } \\
\text { set }(N=71)\end{array}$ & $\begin{array}{l}\text { Per-protocol } \\
\text { set }(N=55)\end{array}$ \\
\hline $\begin{array}{l}\text { Proportion of seizure-free patients }[n(\%)] \\
\text { Seizure-free }\end{array}$ & $71(15.5)$ & $7(12.7)$ \\
$\quad$ Not seizure-free & $60(84.5)$ & $48(87.3)$ \\
Proportion of responders $[n(\%)]$ & & \\
$\quad$ Responder & $39(54.9)$ & $31(56.4)$ \\
Non-responder & $32(45.1)$ & $24(43.6)$ \\
\hline
\end{tabular}

statistically significant $(p=0.2852)$, the mean (SD) overall score improved from 58.5 (14.93) at baseline to 61.3 (17.93) at the last visit. Importantly, the cognitive functioning score did not worsen over the course of treatment (mean change of +1.3 , not significant).
Thirty-three patients $(46.5 \%)$ considered that their intellectual ability was 'very much improved', 'much improved' or 'minimally improved' in relation to baseline. Intellectual ability was considered to have worsened in the PGIC of 12 patients (16.9\%) (see Table 4 in the electronic supplementary material).

\section{Discussion}

This open-label, single-arm, non-controlled trial investigated the safety and efficacy of ESL as adjunctive therapy in patients aged $\geq 65$ years with focal epilepsy over a 26-week treatment period. A total of 72 patients were enrolled to ESL treatment and were analysed for safety and efficacy.

The mean treatment duration was 151.8 days and the mean ESL dose during the overall treatment period was $591.9 \mathrm{mg} /$ day. The overall frequency of TEAEs was $65.3 \%$, 
which is comparable to that reported for the phase III studies in adults with ESL as adjunctive therapy [12-15]. This incidence was lower when compared with oxcarbazepine [16], which reported at least one TEAE for 42 of 52 elderly patients $(81 \%)$, as well as for $78 / 95$ elderly patients $(82 \%)$ exposed to zonisamide [17]. A similar trend was attained for TEAEs at least possibly related in this study $(31 / 72$ patients, $43.1 \%$ ) when compared with that reported for zonisamide (53/95 patients, 55.8\%) [17].

The most common TEAEs in the current trial were dizziness $(12.5 \%)$, somnolence $(9.7 \%)$, fatigue $(8.3 \%)$, convulsion $(8.3 \%)$ and hyponatraemia $(8.3 \%)$. These are known common side effects of ESL, but the incidence of hyponatraemia was higher than that observed in the phase III adjunctive studies (1.5\%) [14]. In fact, a small mean reduction of serum sodium level was found at week 8 in both the ESL $800-\mathrm{mg}$ and ESL $1200-\mathrm{mg}$ groups $(-1.6$ and $-2.2 \mathrm{mEq} / \mathrm{L}$, respectively) compared with placebo $(0.1 \mathrm{mEq} / \mathrm{L})$ [14]. In the present study, of those six subjects with a TEAE of hyponatraemia, one had reduced levels of sodium at baseline, but considered non-clinically significant, and five had normal values of sodium at baseline. Among the five patients with normal sodium levels at baseline, one had an acute episode of chronic gastritis one day after starting ESL treatment, with discontinuation of ESL on day 5. Low sodium level was measured on day 8 . Two patients had hyponatraemia around week 14 of ESL treatment, one at week 6 , and the fourth subject on week 23 of ESL treatment (data not presented in the results section). Despite the elderly population having the highest incidence of hyponatraemia, with higher co-morbidities, and are often medicated with drugs that may themselves lead to hyponatraemia (diuretics), no special concern is given to elderly patients when compared with other aged groups, and no dose adjustment of ESL is needed if the renal function is not disturbed [18].

The majority of TEAEs were of mild or moderate intensity, similar to those reported with other AEDs [16, 17]. Rash was uncommon, seen in two (2.8\%) patients, neither of which were assessed as severe or serious. Three deaths were reported during the study (cardiac failure, glioblastoma multiforme and ischaemic stroke), but none were considered related to ESL treatment, either by the investigator or the sponsor.

The incidences of serious adverse events (SAEs) (13.9\%) and TEAEs leading to study discontinuation $(22.2 \%)$ were higher than that observed for the phase III studies [12-15]. This was expected given the higher incidence of comorbidities in the elderly (as observed in the study). Similar results were reported for zonisamide, with incidence of SAEs and TEAEs leading to discontinuation of $12.6 \%$ and $17.9 \%$, respectively [17], whilst $26.9 \%$ (14/52 patients) discontinued oxcarbazepine due to TEAEs [16]. Moreover, there were no different trends in the types of these events when compared with phase III studies [12-15]: no SAE occurred in more than one subject, and the TEAEs leading to the discontinuation of more than one subject were hyponatraemia ( 3 patients), dizziness and fatigue ( 2 patients each).

Norris' adapted mental sedation scales showed minor changes in subject responses towards a minor worsening of mean values. Slight improvements were observed for all QOLIE-31 scores throughout the treatment period. Importantly, the cognitive functioning score did not worsen over the course of treatment, and rather, was slightly improved at last study assessment. No reports of suicidality (suicidal ideation or behaviour) were observed post-baseline, as assessed by TEAEs and the C-SSRS.

The assessment of efficacy was not a primary objective of the study; nevertheless, it can be observed that the mean standardized seizure frequency decreased from 4.8 during the 8-week baseline period to $3.6(-35 \%)$ during the 26-week treatment period. For other seizure efficacy variables, the proportion of responders and seizure-free patients were $54.9 \%$ and $15.5 \%$, respectively. In a 1-year observational study of add-on levetiracetam in elderly patients with focal epilepsy, the mean monthly seizure frequency decreased from 7.0 at baseline to 1.4 at 12 months, whilst seizure freedom of $58 \%$ at 12 months' follow-up was reported [19]. As described by the authors, a potential confounder of this study was that efficacy analysis was based on the PP population ( $n=364$; safety population, $n=491$ ), which might exclude patients with a negative initial treatment effect and poor tolerance. The mean duration of epilepsy was 13 years (range 0-75), whilst in the present ESL study it was about 21 years (range 1-71). In fact, the elderly patients eligible for this study represent a relatively refractory population since patients with mean epilepsy duration of 246.8 months were under treatment with one or two AEDs and had at least two focal seizures in the 4 weeks prior to enrolment. In clinical practice, most elderly patients respond favourably to monotherapy and rarely need an add-on treatment to control their seizures [3].

The efficacy results were consistent with known previous data on ESL obtained from the phase III pivotal population [14]. With regards to other adjunctive ESL studies, an analysis of patients aged $>65$ years included within the ESLIBASE study showed that, at 6 months, $24.1 \%$ of elderly patients were seizure free, similarly to our findings [20]. It is also similar to a retrospective open-label study of zonisamide as monotherapy/add-on treatment in focal and primary generalized epilepsy stratified by age, where seizure freedom was achieved in $27 \%$ of elderly patients, although $36 \%$ of the elderly epileptic patients received monotherapy, and were less refractory patients [21].

The main difficulty of this study was with patient recruitment, which limited the sample size. Another limitation was 
the open-label, non-controlled and single-arm study design including a refractory population with variable epilepsy duration. Nevertheless, the data presented in this study could be useful for clinicians treating elderly patients with epilepsy as it offers a more realistic picture of everyday practice compared with the rigorous inclusion criteria and too-short follow-up period of controlled trials.

\section{Conclusions}

ESL taken once daily (400-1200 mg) as adjunctive therapy in patients aged $\geq 65$ years was found to be safe, well tolerated and efficacious. The known safety and tolerability profile of ESL was confirmed without any new findings of concern, although higher incidences of hyponatraemia were observed without relationship between its incidence or severity and ESL dose.

\section{Compliance with Ethical Standards}

Funding This study was sponsored by BIAL-Portela \& $\mathrm{C}^{\mathrm{a}}$, S.A.

Conflict of interest All authors were involved in the design or conduct of the study, the collection, management or analysis of the data, and the preparation or review of the manuscript. Drs Costa, Gama, Ikedo, Rocha and Soares-da-Silva are or were employees of BIAL at the time of the study. Dr Steinhoff has no conflicts of interest to declare.

Ethical approval The study was approved by national Independent Ethics Committee and Competent Authorities. The study was conducted according to the Helsinki Declaration, ICH Good Clinical Practice recommendations and applicable local regulations.

Informed consent Written informed consent was received from all subjects prior to the conduct of any trial-related procedures.

Open Access This article is distributed under the terms of the Creative Commons Attribution-NonCommercial 4.0 International License (http://creativecommons.org/licenses/by-nc/4.0/), which permits any noncommercial use, distribution, and reproduction in any medium, provided you give appropriate credit to the original author(s) and the source, provide a link to the Creative Commons license, and indicate if changes were made.

\section{References}

1. CHMP/EWP/566/98 rev 2, Committee for medicinal products for human use. Guideline on clinical investigation of medicinal products in the treatment of epileptic disorders. July $2 \mathrm{http}: / /$ www.ema.europa.eu/docs/en_GB/document_library/Scientific guideline/2010/01/WC500070043.pdf. Accessed 25 Sept 2018.

2. CPMP/ICH/379/95, International Conference of Harmonisation (ICH) E7. Studies in Support of Special Populations: Geriatrics. 1994. http://www.ema.europa.eu/docs/en_GB/document_libra ry/Scientific_guideline/2009/09/WC500002875.pdf. Accessed 25 Sept 2018.
3. Motika PV, Spencer DC. Treatment of epilepsy in the elderly. Curr Neurol Neurosci Rep. 2016;16(11):96.

4. Almeida L, Soares da Silva P. Eslicarbazepine acetate (BIA 2-093). Neurotherapeutics. 2007;4(1):88-96.

5. Bonifacio MJ, Sheridan RD, Parada A, Cunha RA, Patmore L, Soares da Silva P. Interaction of the novel anticonvulsant, BIA 2 093, with voltage-gated sodium channels: comparison with carbamazepine. Epilepsia. 2001;42(5):600-8.

6. Falcão A, Maia J, Almeida L, Mazur D, Gellert M, Soares da Silva P. Effect of gender on the pharmacokinetics of eslicarbazepine acetate (BIA 2 093), a new voltage gated sodium channel blocker. Biopharm Drug Dispos. 2007;28(5):249-56.

7. Almeida L, Falcão A, Maia J, Mazur D, Gellert M, Soares da Silva P. Single dose and steady state pharmacokinetics of eslicarbazepine acetate (BIA 2 093) in healthy elderly and young subjects. J Clin Pharmacol. 2005;45(9):1062-6.

8. Maia J, Almeida L, Falcão A, et al. Effect of renal impairment on the pharmacokinetics of eslicarbazepine acetate. Int J Clin Pharmacol Ther. 2008;46(3):119-30.

9. Maia J, Vaz da Silva M, Almeida L, et al. Effect of food on the pharmacokinetic profile of eslicarbazepine acetate (BIA 2 093). Drugs R.D. 2005;6(4):201-6.

10. Almeida L, Potgieter JH, Maia J, Potgieter MA, Mota F, Soaresda-Silva P. Pharmacokinetics of eslicarbazepine acetate in patients with moderate hepatic impairment. Eur J Clin Pharmacol. 2008;64(3):267-73.

11. Norris $\mathrm{H}$. The action of sedatives on brain stem oculomotor systems in man. Neuropharmacology. 1971;10(21):181-91.

12. Ben-Menachem E, Gabbai AA, Hufnagel A, Maia J, Almeida L, Soares-da-Silva P. Eslicarbazepine acetate as adjunctive therapy in adult patients with partial epilepsy. Epilepsy Res. 2010;89:278-85.

13. Elger C, Halasz P, Maia J, Almeida L, Soares-da-Silva P. Efficacy and safety of eslicarbazepine acetate as adjunctive treatment in adults with refractory partial-onset seizures: a randomized, double-blind, placebo-controlled, parallel-group phase III study. Epilepsia. 2009;50:454-63.

14. Sperling MR, Abou-Khalil B, Harvey J, et al. Eslicarbazepine acetate as adjunctive therapy in patients with uncontrolled partial-onset seizures: results of a phase III, double-blind, randomized, placebo-controlled trial. Epilepsia. 2015;56:244-53.

15. Gil-Nagel A, Lopes-Lima J, Almeida L, Maia J, Soares-da-Silva P. Efficacy and safety of 800 and $1200 \mathrm{mg}$ eslicarbazepine acetate as adjunctive treatment in adults with refractory partialonset seizures. Acta Neurol Scand. 2009;120:281-7.

16. Kutluay E, McCague K, D’Souza J, Beydoun A. Safety and tolerability of oxcarbazepine in elderly patients with epilepsy. Epilepsy Behav. 2003;4(2):175-80.

17. Trinka E, Giorgi L, Patten A, Segieth J. Safety and tolerability of zonisamide in elderly patients with epilepsy. Acta Neurol Scand. 2013;128(6):422-8.

18. European Medicines Agency. Zebinix (eslicarbazepine acetate): EU summary of product characteristics. 2017. https://www.ema. europa.eu/documents/product-information/zebinix-epar-produ ct-information_en.pdf. Accessed 29 Sept 2018.

19. Werhahn KJ, Klimpe S, Balkaya S, Trinka E, Krämer G. The safety and efficacy of add-on levetiracetam in elderly patients with focal epilepsy: a one-year observational study. Seizure. 2011;20(4):305-11.

20. Gómez-Ibáñez A, Serratosa JM, Guillamón E, et al. Efficacy and safety of eslicarbazepine-acetate in elderly patients with focal epilepsy: Case series. Seizure. 2017;48:53-6.

21. Ramsay RE. Zonisamide (ZNS, Zonegran (R)): efficacy and safety stratified by patient age, p292. abstracts from the 25 th international epilepsy congress Lisbon, Portugal October 12-16, 2003. Epilepsia. 2003;44:1-223. 


\section{Affiliations}

\section{Raquel Costa ${ }^{1} \cdot$ Bernhard Steinhoff $^{2} \cdot$ Helena Gama ${ }^{1} \cdot$ Fábio Ikedo $^{1} \cdot$ José-Francisco Rocha ${ }^{1}$.}

Patrício Soares-da-Silva ${ }^{1,3,4}$

1 Department of Research and Development, BIAL-

Portela \& C $C^{a}$, S.A., À Avenida da Siderurgia Nacional, 4745-457 Coronado (S. Romão e S. Mamede), Portugal

2 Epilepsy Center Kork, Landstrasse 1, 77694 Kehl, Germany
3 Department of Biomedicine, Unit of Pharmacology and Therapeutics, Faculty of Medicine, University of Porto, Porto, Portugal

4 MedInUP-Center for Drug Discovery and Innovative Medicines, University of Porto, Porto, Portugal 